\title{
COMS Normal Operation for Earth Observation Mission Young-Min Cho
}

\author{
Geostationary Satellite Operation Team, Korea Aerospace Research Institute
}

\begin{abstract}
Communication Ocean Meteorological Satellite (COMS) for the hybrid mission of meteorological observation, ocean monitoring, and telecommunication service was launched onto Geostationary Earth Orbit on June 27,2010 and it is currently under normal operation service on $128.2^{\circ}$ East of the geostationary orbit since April 2011. In order to perform the three missions, the COMS has 3 separate payloads, the meteorological imager (MI), the Geostationary Ocean Color Imager (GOCI), and the Ka-band antenna. The MI and GOCI perform the Earth observation mission of meteorological observation and ocean monitoring, respectively. For this Earth observation mission the COMS requires daily mission commands from the satellite control ground station and daily mission is affected by the satellite control activities. For this reason daily mission planning is required. The Earth observation mission operation of COMS is described in aspects of mission operation characteristics and mission planning for the normal operation services of meteorological observation and ocean monitoring. And the first one-year normal operation results after the In-Orbit-Test (IOT) are investigated through statistical approach to provide the achieved COMS normal operation status for the Earth observation mission.
\end{abstract}

Key Words : Communication Ocean Meteorological Satellite (COMS), MI, GOCI, Earth observation, satellite operation, mission planning, satellite image reception

\section{Introduction}

Communication Ocean Meteorological Satellite (COMS) for the hybrid mission of meteorological observation, ocean monitoring, and telecommunication service has been launched onto Geostationary Earth Orbit on 27 June 2010 (Korea Standard Time: KST). After launch the COMS has been located at the altitude of $35,800 \mathrm{~km}$ on the $128.2^{\circ}$ East of the Equator, geostationary Earth orbit. After successful completion of the In-Orbit-Test (IOT) for about 7 months, the COMS is currently under the normal operation for the regular mission services of meteorological observation, ocean monitoring, and telecommunication. The COMS has three payloads, which are the Meteorological Imager (MI), the Geostationary Ocean Color Imager (GOCI), and the Ka-band antenna for the three missions (Cho and Youn, 2006; Kim et al., 2009; Lee et al., 2010a). Each payload performs its dedicated mission respectively. The Earth observation mission

Received May 23, 2013; Revised June 18, 2013; Accepted June 28, 2013.

Corresponding Author: Young-Min Cho (ymcho@kari.re.kr)

This is an Open-Access article distributed under the terms of the Creative Commons Attribution Non-Commercial License (http:/creativecommons.org/ licenses/ by-nc/3.0) which permits unrestricted non-commercial use, distribution, and reproduction in any medium, provided the original work is properly cited 
operation of meteorological observation and ocean monitoring is performed by the MI and the GOCI respectively. Unlike telecommunication mission which is operated under long term control by ground station, the Earth observation mission requires daily mission commands from the satellite control ground station and daily mission is affected by the satellite control activities. For this reason Earth observation mission planning is carried out daily based on user mission request.

During the IOT phase the functionality and the performance of many aspects of the COMS satellite and ground station had been checked and tested through the Earth observation mission operation of meteorological observation and ocean monitoring (Kim et al., 2010; Lee et al., 2011a). The mission operation results during the COMS IOT were reported through statistical approach for the study of the mission operation capability of COMS achieved and verified during the IOT and the satellite image reception status during the IOT (Cho, 2013). In this report, during the IOT it was tested that the COMS meteorological observation mission was operated successfully with the Earth imaging operation rate of $80 \%$ of 24 hours a day over 3 months with respect to the IOT normal meteorological mission schedule. Also the COMS ocean monitoring mission was operated 8 times a day with the ocean imaging operation rate of $17 \%$ during the IOT.

Since April 2011, the COMS Earth observation mission is under the normal operation 24 hours a day 365 days a year. The normal operation of the COMS is conducted by the Satellite Ground Control System (SGCS) in the Satellite Operation Center (SOC) of the Korea Aerospace Research Institute (KARI). The SGCS consists of the Flight Dynamic Subsystem (FDS), the Mission Planning Subsystem (MPS), the Real-Time Operation Subsystem (ROS), and the Telemetry, and Tracking \& Command (TT\&C) including the ground antenna (Lee et al., 2011a; Cho et al., 2012). For the mission planning of the COMS normal operation, the MPS synthesizes the user mission requests for 'meteorological observation and ocean monitoring' and 'the satellite orbit and attitude control mission', resolves conflicts between missions, and establishes daily mission plan of the Earth observation and the satellite control. In this paper, the mission operation characteristics of meteorological observation and ocean monitoring for the normal operation services are discussed in the point of overview. And the discussion deals with daily mission planning characteristics. The first one-year normal operation results are analyzed through statistical approach to provide the mission operation status of COMS and the satellite image reception status which are achieved during the first one-year normal operation from April 2011 to March 2012.

Please note that this paper provides achieved normal mission operation capability of the COMS which performs the first ocean monitoring mission in the world, the first meteorological observation mission in Korea, and the first simultaneous operation of meteorological and ocean mission on the geostationary Earth orbit.

\section{Meteorological Mission Operation Characteristics}

The MI has 5 spectral channels in the visible and infrared (IR) region (1 visible channel and 4 IR channels) and the spatial resolution of the MI is $1 \mathrm{~km}$ at nadir for the visible channel and $4 \mathrm{~km}$ at nadir for all the IR channels (Cho and Youn, 2006). The MI is required to operate its mission continuously through normal Earth imaging and in-orbit calibration with minimum performance degradation during the satellite eclipse periods. Also the user requirements define the three observation modes of global, regional, and local mode as well as the five MI observation areas of FD, 
Table 1. MI Observation Area and Image Number

\begin{tabular}{|c|c|c|c|}
\hline $\begin{array}{l}\text { Observation } \\
\text { Mode }\end{array}$ & Observation Area & Field Of View $(\mathrm{FOV})^{*}$ Requirement & $\begin{array}{l}\text { The number of images for the daily } \\
\text { requested normal operation } \\
\text { meteorological schedule }\end{array}$ \\
\hline Global & Full Disc (FD) & $\begin{array}{l}E W: \geq 19^{\circ} \\
N S: \geq 17.6^{\circ}\end{array}$ & ( \\
\hline \multirow{3}{*}{ Regional } & $\begin{array}{l}\text { Asia and Pacific in North } \\
\text { Hemisphere (APNH) }\end{array}$ & $\begin{array}{l}\text { EW: }-2.2^{\circ} \sim+4.3^{\circ} \\
\text { NS: }+8.1^{\circ} \sim+3.2^{\circ}\end{array}$ & 0 \\
\hline & $\begin{array}{c}\text { Extended North } \\
\text { Hemisphere (ENH) }\end{array}$ & $\begin{array}{l}\text { EW: }-6.2^{\circ} \sim+6.2^{\circ} \\
\text { NS: }+8.1^{\circ} \sim-1.8^{\circ}\end{array}$ & 80 \\
\hline & $\begin{array}{l}\text { Limited Southern } \\
\text { Hemisphere (LSH) }\end{array}$ & $\begin{array}{l}\text { EW: }-6.2^{\circ} \sim+6.2^{\circ} \\
\text { NS: }-1.8^{\circ} \sim-6.9^{\circ}\end{array}$ & 0 \\
\hline Local & Local Area (LA) & $\begin{array}{l}\text { EW: } 1000 \mathrm{~km} \\
\text { NS: } 1000 \mathrm{~km} \\
\text { (Random selection in the FD area)** }\end{array}$ & 88 \\
\hline
\end{tabular}

* FOV Center: the Satellite Nadir Point of $128.2^{\circ}$ East, EW: East-West, NS: North-South

** In the normal operation of COMS, the LA image is taken around the Korean peninsula.

APNH, ENH, LSH, and LA as defined and shown in the Table 1 and the Fig. 1. The operational imaging areas of the 5 images include some marginal area to take care of the pointing errors due to the satellite orbit maintenance, attitude control, instrument alignment, and so on. So that operational areas are a little larger than the requirements of the Table 1.

During the IOT of the COMS, the MI images of all the 5 observation areas were taken for functionality and performance test. However in the normal operation of the COMS, the meteorological mission is performed for the 3 observation areas of FD, ENH, and LA only and the imaging area of LA is fixed around the Korean peninsula, according to the change request by user in order to have more observations on the meteorological phenomena around the Northern hemisphere of the Earth and the Korean peninsula.

In general the meteorological mission schedule for the COMS operation is planned one day early before the local date of the mission execution. The missions for 30 minutes are used as a building block for mission planning of the Earth observation of the COMS. The mission planning is performed by arranging the missions by 30 minutes properly during the time zone of the mission execution (normally 24 hours). The unit mission slot of 30 minutes used for meteorological

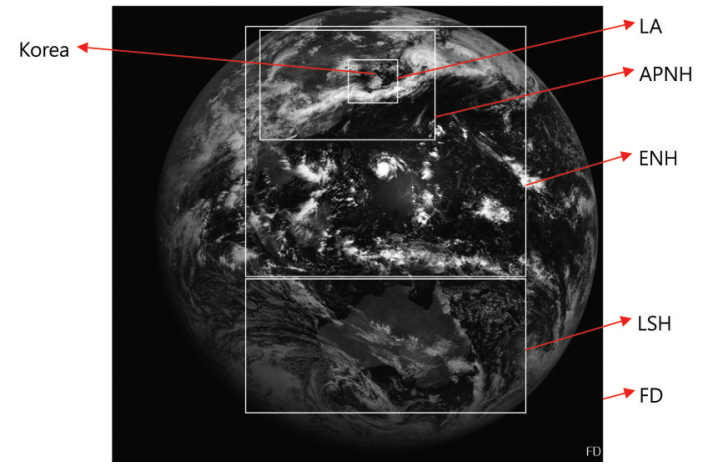

Fig. 1. The Typical Meteorological Image Areas

mission planning is called the MI sequence. Every MI sequence begins with a Blackbody Calibration (BBCAL) followed by meteorological images within 30 minutes. So the time interval between the start time of the BBCAL and the end time of the last image of the sequence should be within 30 minutes. And the BBCAL usually performs every 30 minutes and meteorological images are taken between the BBCALs (Cho and Youn, 2006).

The normal operation of the meteorological observation mission is executed under the control of the KARI SOC and the meteorological images are distributed officially by the National Meteorological Satellite Center (NMSC) of Korea Meteorological Administration (KMA) since April 1, 2011. In the normal operation, it is requested based on the user requirement that the global mode combined with the 
local mode is executed every 3 hours and the regional observation mode combined with the local mode are evoked for 2hours and 30 minutes between two FD images which are separated 3 hours. The MI sequence for the combined global observation mode consists of the two images of "FD+LA" and the MI sequence for the combined regional observation mode is comprised of the four images of "ENH+LA+ENH+LA". Please note that the combined regional mode of the normal operation is changed from the five images of "ENH+LA+APNH+LSH+LA" of the IOT normal meteorological mission schedule (Cho, 2013). In addition to the Earth imaging, in the normal operation there are 3 special observation missions for support of meteorological observation missions. The Albedo Monitoring is performed to have the Sun image once a day (about 21:30 Universal Time Coordinated: UTC) for the check of the responsivity of the MI visible channel. The Moon observation and dark space observation are carried out monthly and quarterly respectively in order to check of the variation of the MI radiometric performance.

In the normal operation, the NMSC of KMA requests the KARI SOC the daily user request of the normal operation meteorological mission schedule which is packed with only the Earth observation without any special observation mission such as Albedo Monitoring, Moon, and dark space observation. Since the FD image is taken every 3 hours, the number of the FD images is 8 a day. The combined regional modes repeat 5 times between the FD images so that the ENH images are taken 80 times a day. The number of LA images is 88 a day because they are included in both the combined global mode and the combined regional modes. The number of images for the daily user request of the normal operation meteorological mission schedule is summarized in the Table 1 . The daily requested normal operation meteorological schedule is also shown in the Fig. 2 in terms of the ratio of accumulated mission duration to 24 hours a day,

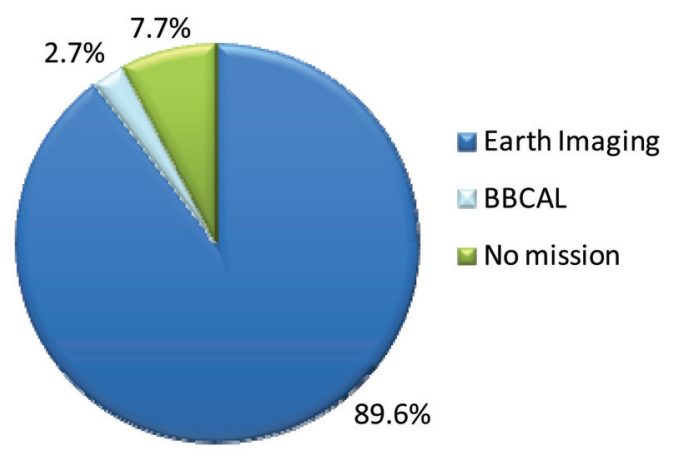

Fig. 2. The daily mission duration ratio of the requested meteorological schedule for COMS normal operation (theoretical limit)

where the duration ratio of Earth imaging is for all the images to be taken a day and the duration ratio of BBCAL is for all the 48 BBCALs a day. The mission duration ratio of the Fig. 2 is the maximum allowable value which can be achieved theoretically in the normal operation meteorological mission schedule. So, the daily total meteorological mission is requested to be performed during $92.3 \%$ of 24 hours a day, which is theoretical limit and the sum of the Earth imaging of $89.6 \%$ and the BBCAL of $2.7 \%$. It is noteworthy that the user request of the normal operation meteorological schedule requires about 5\% more Earth imaging time than that of the IOT normal meteorological mission schedule which has the Earth imaging duration ratio of $84 \%$ (Cho, 2013). There is no change of the BBCAL schedule between the user requests of the normal operation meteorological schedule and the IOT normal meteorological mission schedule.

\section{Ocean Mission Operation Characteristics}

The GOCI has 8 spectral channels in the visible and near IR region of the light spectrum and the spatial resolution of the GOCI is $500 \mathrm{~m}$ at the center of the observation area for all the channels (Kim et al.,2009). The required observation area of the GOCI image is fixed with the size of $2500 \times 2500 \mathrm{~km}$ around the 


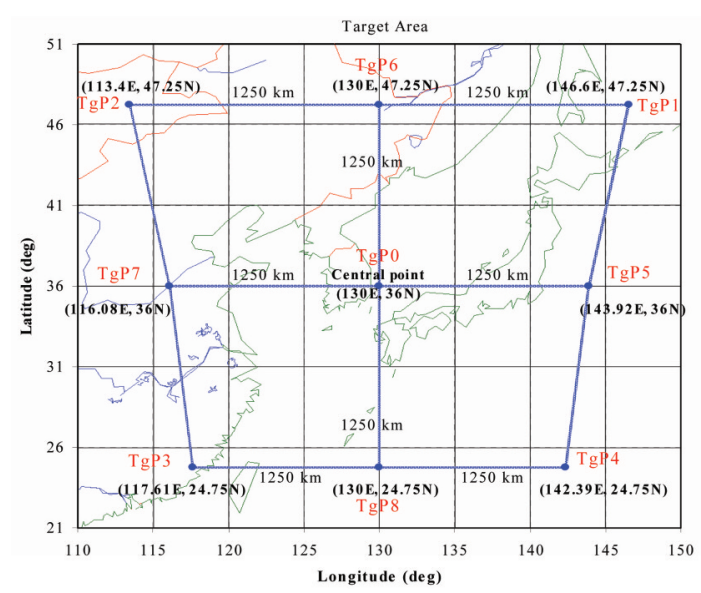

Fig. 3. The required GOCI Image Area

Korean peninsula (See the Fig. 3). The operational imaging areas of the GOCI image include some marginal area to take care of the pointing errors due to the satellite orbit maintenance, attitude control, instrument alignment, and so on. So that operational areas are a little larger than the area of the Fig. 3. It takes about 30 minutes to observe this area one time by the GOCI. The ocean monitoring mission of the COMS is performed one time every hour from 00:15 (UTC) to have 8 times observations a day so that ocean images around the Korea peninsular are taken during daytime (Korean local time). In addition to ocean monitoring, in-orbit calibration of the GOCI is performed at local night time periodically (Faure et al., 2012).

Since the Korea Ocean Satellite Center (KOSC) of Korea Institute of Ocean Science \& Technology (KIOST) distributes officially from April 20, 2011 the COMS ocean images which have been taken under the control of the KARI SOC since April 1, 2011, the ocean mission of the COMS is assumed to be under normal operation since April 1, 2011. Similarly to the meteorological observation mission, the ocean mission schedule for the COMS operation is planned one day early before the local date of the mission execution and the missions for 30 minutes are used as a building block for mission planning of the Earth observation of the COMS. The mission planning is performed by

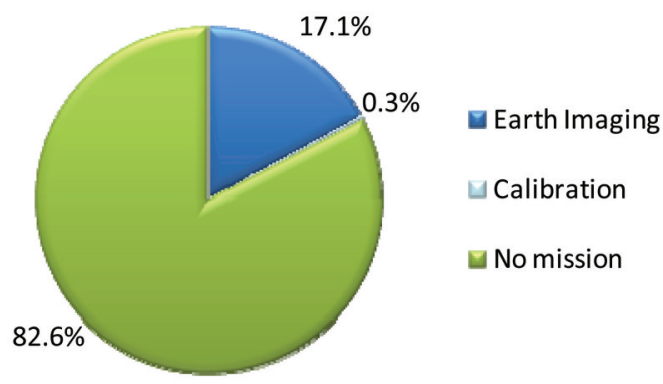

Fig. 4. The daily mission duration ratio of the requested ocean mission schedule for COMS normal operation (theoretical limit)

arranging the missions by 30 minutes properly during the time zone of the mission execution. Since it takes about 30 minutes to complete the ocean monitoring mission one time, an ocean image is used as the unit mission slot of 30 minutes for ocean mission planning in itself.

The daily user request for the ocean mission schedule of COMS normal operation is shown in the Fig. 4 in terms of the ratio of accumulated mission duration to 24 hours a day, where the duration ratio of Earth imaging is for all the ocean images to be taken a day and the duration ratio of calibration is for the inorbit calibration of the GOCI a day. The mission duration ratio of the Fig. 4 is also the maximum allowable value which can be achieved theoretically in the ocean mission schedule. So, the daily total ocean mission is requested to be performed during $17.4 \%$ of 24 hours a day, up to theoretical limit, which is the sum of the Earth imaging of $17.1 \%$ and the calibration of $0.3 \%$. Since the GOCI calibration has been fully characterized during the COMS IOT, in the COMS normal operation the GOCI calibration is performed less frequently up to almost half of the number of the COMS IOT calibration. So that the daily mission duration ratio of the GOCI calibration for the COMS normal operation is reduced to almost half of the COMS IOT calibration rate $(0.6 \%$; Cho, 2013). Since the ocean imaging schedule of the normal operation is not changed from that of the IOT, the Earth imaging 
duration ratio of the ocean mission normal operation is the same as that of the IOT.

\section{Mission Planning for the Earth Observation}

In the normal operation of the COMS, the MPS is to plan the operational schedule of the Earth observation mission. The MPS consists of three functional parts, Mission Request Gathering, Mission Scheduling, and Mission Schedule Reporting. First, the Mission Request Gathering collects the mission requests for 'meteorological observation and ocean monitoring' from the users and the mission requests for satellite orbit and attitude control from the FDS, to process them to the data with the format suitable to build a schedule. Next, the Mission Scheduling builds the mission schedule from the processed data of the mission request to provide the display of the mission schedule, the check of schedule constraints, the check of conflicts among missions, the resolution of the conflicts, the generation of the final mission schedule files, the generation \& the delivery of the mission schedule command files for sending to the satellite, the generation of the event files for the image data processing, and the management of the mission planning files. The MPS receives additional information related to the satellite orbit, the Sun, and the Moon from the FDS. Based on the user mission requests and the FDS satellite control requests, the MPS plans mission schedules, generates the mission schedule commands, and sends them to the ROS for upload to the satellite. Finally, the Mission Schedule Reporting sends the mission planning results, which are the mission schedule and the event files for the image data processing, to the users of the Earth observation image data.

There are some constraints on the Earth observation due to the management of the satellite in the normal operation of the COMS. In order to meet the operational requirement on the satellite attitude and orbit maintenance, the Wheel Off Loading (WOL) maneuver of the satellite is performed twice a day (Lee et al., 2006; Park et al., 2006) and the Station Keeping (SK) maneuver of the satellite is carried out once a week in the East-West direction and in the North-South direction, respectively (Lee et al., 2010b; Lee et al., 2011b). Thanks to the SK maneuvers, the satellite orbit maintenance is kept at the $128.2^{\circ}$ East of the Equator within the accuracy of $+/-0.05^{\circ}$ in the East-West direction and in the North-South direction, respectively. The MPS receives the mission request for WOL and SK with their schedule information from the FDS. In the case the meteorological observation mission or the ocean monitoring mission conflicts with the WOL or the SK, the conflict is resolved in the mission planning process normally as per the COMS mission priority rule and the mission conflict management specifications. As the result of the conflict management, lower priority missions can be removed or moved to another time in the daily mission schedule.

A daily (24 hours) mission schedule of the COMS Earth observation is shown in the Fig. 5 for the example of the normal operation of the COMS. The Fig. 5 shows the meteorological mission schedule and the ocean mission schedule as well as the two WOLs, one SK, and one Albedo Monitoring. In the Fig. 5 for the satellite attitude and orbit control by the FDS the two WOLs occur at 00:45 (UTC) and 15:21 (UTC) respectively and the SK takes place at around 20 o'clock (UTC). Since these two WOLs and one SK should be performed simultaneously with some meteorological missions of the requested meteorological mission schedule, there are mission conflicts, which need to be resolved by the MPS. After the mission conflict management applied, some meteorological missions are not planned in order to avoid confliction with the satellite maneuvers. Two ENH image missions are not planned due to the two WOLs and two ENH image missions are canceled due to the SK. The next 


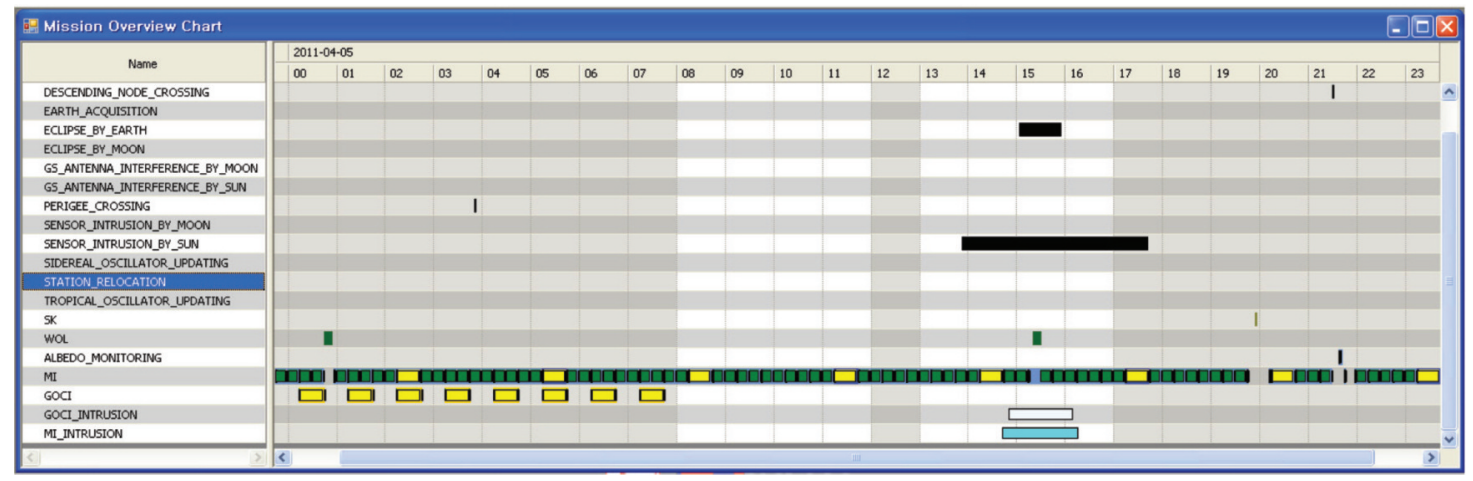

Fig. 5. A daily mission schedule of the Earth observation for the COMS normal operation

mission conflict management is to give up two ENH image missions for the Albedo Monitoring which performs at 21:39 (UTC). So, the total image number of the normal operation meteorological mission is reduced from 176 of the requested schedule (FD:8, ENH: 80, LA: 88) to 171 of the operated schedule (FD:8, ENH: 74,LA: 88, Albedo Monitoring: 1) in the Fig. 5.

\section{Mission Operation Results for the First One-Year Normal Operation}

The normal operation of the COMS Earth observation mission is performed by the cooperation of the NMSC of KMA, the KOSC of KIOST, and the SOC of KARI. In the normal operation, the NMSC provides the meteorological mission request and takes the primary role for the reception, preprocessing, postprocessing, archive, distribution (satellite broadcast and ground network), and application of the meteorological image data. And the KOSC provides the ocean mission request and takes the primary role for the reception, preprocessing, postprocessing, archive, distribution, and application of the ocean image data. The KARI SOC takes the responsibility for the satellite control including mission planning of Earth observation and provides the backup of the reception, preprocessing, archive, and satellite broadcast distribution of both the meteorological image data and the ocean image data of the COMS.

In this paper, the Earth observation mission operation during the first year normal operation is analyzed and synthesized through statistical approach with the COMS mission planning results and the satellite image reception status which are the outcome of the COMS mission operation. The satellite image reception status can be different slightly between primary and backup ground station depending on daily operational status of the station. In this paper the image reception status is presented for only the KARI SOC.

The meteorological mission schedules of the first one-year normal operation from April 2011 to March 2012 (12 months) have been investigated to obtain the average values of daily satellite operation rate for the meteorological mission operation modes of Earth Imaging, Non-Earth Imaging, Standby, and Maneuver, which are introduced simply for easy understanding of the meteorological mission operation status of the COMS in this paper and defined as the following. The Earth Imaging mode is the operation status that the COMS performs meteorological observation with the Earth scanned by the MI and one of the MI images of $\mathrm{FD}, \mathrm{ENH}$, and LA is being taken by the COMS. The Non-Earth Imaging mode is the operation status that the COMS is operating to observe anything except the Earth by scanning the outside of the Earth with the MI for the support of the meteorological observation of the 
Earth such as the BBCAL and the 3 special observation missions of the Albedo monitoring, the moon observation, and the dark space observation. The Standby mode is the operation status that the COMS is waiting for spacecraft maneuver or imaging 'the Earth or anything else' with the MI put on standby. Note that in this mode the COMS performs most of the spacecraft on-board command processing to execute the MI imaging, the movement between the MI images, and satellite maneuver. The Maneuver mode is the operation status that the COMS performed the WOL or the SK for the satellite attitude and orbit maintenance. The MI imaging duration should be out of the maneuver conflict time slot including the duration of maneuver execution in order to avoid any possible conflict between the MI imaging mission with the maneuver. In this paper, the Maneuver mode has slightly different definition from that of the previous paper by Cho (2013). The Maneuver mode of the COMS normal operation does not include the time gap of the standby mode between the maneuver conflict time slot and the adjacent meteorological missions which was included into that of the COMS IOT operation in the reference of (Cho, 2013), so that the definition has more fidelity to its name in the view point of the satellite control operation instead of the meteorological mission availability. The daily satellite operation rate is the ratio of the total time consumed by the operation mode with respect to the 24 hours of a day. Also the daily satellite image reception status has been monitored in the KARI SOC during the first year normal operation. The satellite image reception status of the first year normal operation can be summarized by using the daily reception number rate of each MI image which is the ratio of the received image number of each MI image (FD, ENH, or LA) with respect to the total received Earth image number of a day.

The averaged daily satellite operation rate of the first year normal operation was obtained by using the meteorological mission planning results of the COMS
MPS for 12 months from April 1, 2011 to March 31, 2012 (See the Fig. 6 (a)). During the first year normal operation of the 12 months, the planning and the execution of meteorological mission were successfully performed without any mission operation failure. It is found in the Fig. 6 (a) that the COMS meteorological Earth observation mission was operated successfully with the Earth imaging operation rate of $86.4 \%$ of 24 hours a day on average during the first year normal operation. It took $3.0 \%$ of 24 hours a day for the NonEarth Imaging, 1.5\% for Maneuver. And the COMS was in the Standby during $9.1 \%$ of 24 hours a day. It took $89.4 \%$ of 24 hours a day for COMS to perform the meteorological observation mission which consists of the Earth Imaging and the Non-Earth Imaging.

According to the daily requested mission duration of the Fig. 2, the theoretically maximum allowable satellite operation rate of the normal operation meteorological mission is $92.3 \%$ a day. This value is compared with the achieved value of $89.4 \%$ of the Fig. 6 (a). This comparison gives that the meteorological mission request is made up to the theoretical limit and the $96.8 \%$ of all the requested meteorological missions have been operated by the COMS except for the missions which are not planned due to confliction with the satellite maneuvers and the 3 special observation missions of the Albedo monitoring, the moon observation, and the dark space observation. In the example of the Fig. 5 where the 6 ENH missions were not planned due to mission confliction, the satellite operation rate of the meteorological mission is reduced by $4.8 \%$, from the limit of $92.3 \%$ to the operated value of $87.5 \%$, which is worse than the one-year average (89.4\% of the Fig. 6 (a)). In average during the normal operation $3 \mathrm{ENH}$ missions are not planned daily due to mission confliction with the two WOLs and the Albedo monitoring and $2 \mathrm{ENH}$ missions are not planned weekly due to mission confliction with the two SKs, by a simple calculation this leads to the missionimpossible time slot of $3.3(=3+2 / 7)$ times the one 


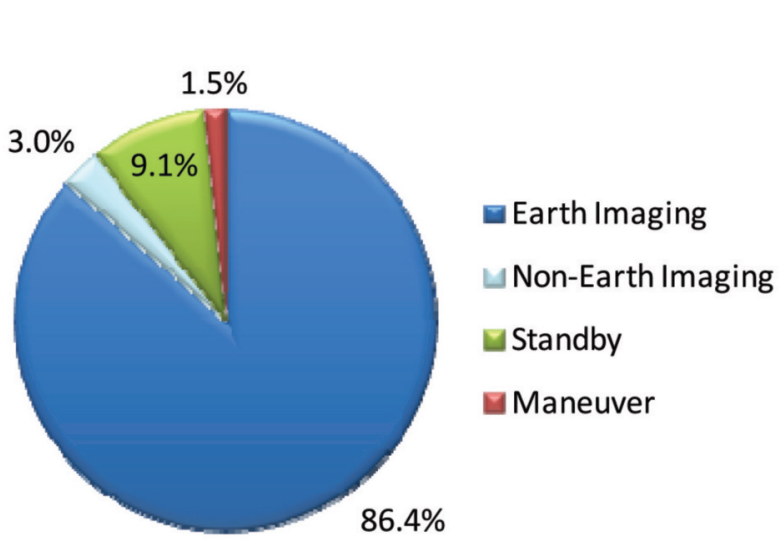

(a) Daily satellite operation rate

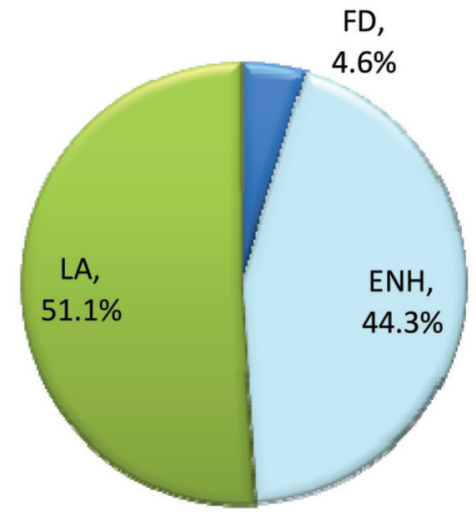

(b) Daily image reception number rate

Fig. 6. The satellite operation status and the Earth observation image reception status of the meteorological mission for COMS normal operation

ENH duration' in a day which approximately corresponds to the reduction by $2.9 \%$ in the daily satellite operation rate of the meteorological mission, from the limit of $92.3 \%$ (the Fig. 2) to the achieved value of $89.4 \%$ (the Fig. 6 (a)). This means that all the COMS meteorological observation missions were operated successfully 24 hours a day over one year with the normal meteorological mission schedule, which is a good verification of the stability and the robustness of the meteorological mission operation on a top system level in all aspects of the satellite bus/payload, the ground station facilities/equipments, and the ground operator activities.

It is noted that the first year normal operation has the Earth imaging operation rate increased by about $6.4 \%$ comparing with the IOT normal meteorological mission operation (Cho, 2013), which has the Earth imaging operation rate of $80 \%$. The reason for this increase is that the imaging duration of the combined regional mode has increased due to the change of the mission schedule from the five images of "ENH+LA $+\mathrm{APNH}+\mathrm{LSH}+\mathrm{LA}$ " to the four images of "ENH+LA+ ENH+LA". The imaging duration increase from the five images to the four images can be understood easily by the calculation with the observation area size of the Table 1. It is found in the Table 1 that the observation area of ENH is larger than the sum of the two areas of APNH and LSH. So that, for the requirements of the observation area, the total area of the four images is about 1.12 times larger than that of the five images. The Maneuver operation rate of the COMS normal operation is $1.5 \%$, which is much less than that of the COMS IOT (4\%). The main reason for this rate difference is the difference of the Maneuver mode definition between the normal operation and the IOT operation as explained in the above.

The average values of daily reception number rate of FD, ENH, and LA during the first year normal operation of the 12 months are shown in the Fig. 6 (b). All the LA images were taken for the Korean peninsula. The daily image reception number rate of the Fig.6 (b) is based on the image reception results of the KARI SOC for the first year normal operation. Among the total Earth images which were taken with the satellite operation rate of $86.4 \%$ and received successfully by the ground station a day, the number of FD images is $4.6 \%$ of the number of total Earth images, the ENH is $44.3 \%$, and the LA is $51.1 \%$. Since the most (more than 95\%) of daily Earth images are the ENH or the LA, it is clear that the meteorological phenomenon around the Korean peninsula has been monitored intensively by the COMS, which is one of key purposes of the COMS 
meteorological mission. Therefore, in point of operation rate and stability on the meteorological mission it is clear that the normal operation service of the COMS has been successful.

The ocean monitoring mission operation of the COMS is much simpler than the meteorological observation mission operation. The ocean monitoring mission is fulfilled by the daytime ocean imaging and the GOCI calibration. Similarly to the meteorological observation mission, the ocean monitoring mission schedules of the first year normal operation have been investigated to obtain the average values of daily satellite operation rate for the ocean mission operation modes of Earth Imaging, Calibration, Standby, and Maneuver, which are introduced simply for easy understanding of the ocean mission operation status of the COMS in this paper and defined as the following. The Earth Imaging mode is the operation status that the COMS performs ocean monitoring with the Earth imaged by the GOCI. The Calibration mode is the operation status that the COMS is operating to calibrate the GOCI. The Standby mode is the operation status that the COMS is waiting for spacecraft maneuver, imaging the Earth, or calibration. The Maneuver mode is the operation status that the COMS performs the North-South SK maneuver for the satellite orbit maintenance. The GOCI imaging duration should be out of the maneuver conflict time slot including the duration of maneuver execution in order to avoid any possible conflict between the GOCI imaging mission with the maneuver. The Maneuver mode of the COMS normal operation does not include the time gap of the standby mode between the maneuver conflict time slot and the adjacent ocean missions which was included into that of the COMS IOT operation in the reference of (Cho, 2013). Only the North-South SK is taken into account in the Maneuver mode of the ocean mission operation because the WOL and the East-South SK are always scheduled to perform outside of the GOCI imaging. The daily satellite operation rate is the ratio of the total time consumed by the operation mode with respect to the 24 hours of a day. The daily satellite operation rate is obtained from the ocean mission planning results of the COMS MPS and the execution results of the mission command sent by the COMS ROS.

The Fig. 7 shows the averaged daily satellite operation rate of the ocean mission during the first oneyear normal operation (12 months) from April 1, 2011 to March 31, 2012. During the first one-year normal operation of the 12 months, the ocean mission planning and mission execution were successfully performed without any mission operation failure. Since the daily satellite operation rate of the Earth (ocean) imaging is $17.0 \%$ of $24 \mathrm{hrs}$ a day in the Fig. 7 , it is confirmed that the COMS ocean monitoring mission was operated successfully 8 times a day during the first one-year normal operation. It took $0.3 \%$ of 24 hours a day for the Calibration, $0.02 \%$ for Maneuver. And the COMS was in the Standby during $82.6 \%$ of 24 hours a day. It took $17.3 \%$ of 24 hours a day for COMS to perform the ocean monitoring mission which consists of the Earth Imaging and the Calibration.

According to the daily requested mission duration of the Fig. 4, the theoretically maximum allowable satellite operation rate of the ocean mission is $17.4 \%$ a day. This value is compared with the achieved value of $17.3 \%$ of the Fig. 7. This comparison gives that the ocean mission request is made up to the theoretical limit and the $99.6 \%$ of all the requested ocean missions have been operated by the COMS except for the missions which are not planned due to confliction with the satellite maneuvers. During the first one-year normal operation, 12 ocean imaging missions were not planned due to mission confliction with the SK, by a simple calculation this leads to the mission-impossible time slot of ' $0.033(=12 / 366)$ times the one ocean imaging duration' which approximately to one decimal place in percent corresponds to the reduction by about $0.1 \%$ in the daily satellite operation rate of the ocean mission, 


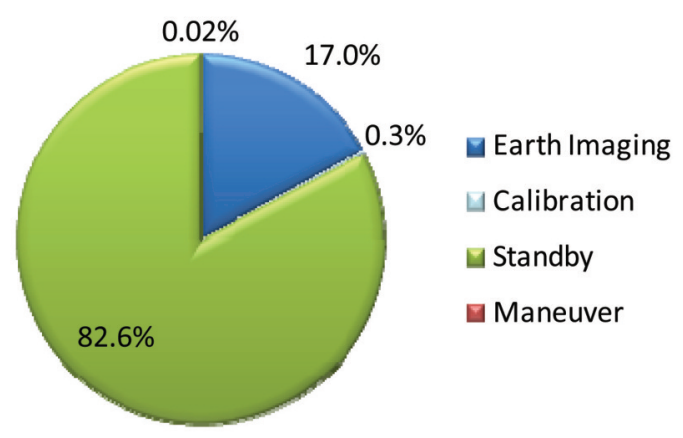

Fig. 7. The daily satellite operation status of the ocean monitoring mission for COMS normal operation

from the limit of $17.4 \%$ (the Fig. 4) to the achieved value of $17.3 \%$ (the Fig. 7). This means that all the COMS ocean observation missions were operated successfully 8 times a day over one year with the normal ocean mission schedule, which is also a good verification of the stability and the robustness of the ocean mission operation on a top system level in all aspects of the satellite bus/payload, the ground station facilities/equipments, and the ground operator activities.

Since ocean images are taken in the same operation condition except for imaging time, they are all the same kind and there is no need to look into the composition of the received images in the point of satellite mission operation, unlike the MI images.

As mentioned above, all the requested schedules of both meteorological and ocean missions have been operated by the COMS except for the missions conflicted with the satellite maneuvers. This reveals that there was no interference between meteorological and ocean mission each other even though simultaneous operation of meteorological and ocean mission was performed.

\section{Conclusions}

Communication Ocean Meteorological Satellite (COMS) for the hybrid mission of meteorological observation, ocean monitoring, and telecommunication service is currently under normal operation service since April, 2011. The meteorological imager (MI) and the Geostationary Ocean Color Imager (GOCI) perform the Earth observation mission of meteorological observation and ocean monitoring, respectively.

The mission operation characteristics of the COMS normal operation is introduced for the Earth observation of meteorological observation and ocean monitoring respectively. The normal operation schedule of the meteorological mission is comprised of the combined global mode of "FD+LA" every 3 hours and the combined regional modes of "ENH+LA+ ENH+LA" between the global modes. In the COMS normal operation, the ocean monitoring mission is performed one time every hour from 00:15 (UTC) to have 8 times observations during daytime (Korean local time) a day.

The COMS mission planning of the COMS normal operation for the Earth observation is described in the point of overview. The MPS synthesizes the user mission requests for 'meteorological observation and ocean monitoring' and 'the satellite orbit and attitude control mission' to resolve conflicts among missions and to establish daily mission schedule of the Earth observation and the satellite control.

The first one-year normal operation results after the In-Orbit-Test (IOT) are investigated through statistical approach to provide the COMS normal operation status achieved for the Earth observation mission during first year normal operation from April 2011 to March 2012 (12 months). The meteorological mission schedules of the first year normal operation have been analyzed to obtain the average values of daily satellite operation rate for the meteorological mission operation modes of Earth Imaging, Non-Earth Imaging, Standby, and Maneuver. Also the satellite image reception status of the first year normal operation is summarized by using the daily reception number rate of each $\mathrm{MI}$ image. The ocean monitoring mission schedules of the first oneyear normal operation have been investigated to obtain 
the average values of daily satellite operation rate for the ocean mission operation modes of Earth Imaging, Calibration, Standby, and Maneuver. The COMS meteorological observation mission was operated successfully with the Earth imaging operation rate of $86.4 \%$ of 24 hours a day on average during the first year normal operation. For the ocean mission, the daily satellite operation rate of the Earth imaging is $17.0 \%$ of $24 \mathrm{hrs}$ a day. The achieved satellite operation rates of the meteorological and ocean missions are $89.4 \%$ and $17.3 \%$ of 24 hours a day, respectively.

As a conclusion, the COMS mission operation results during the first year normal operation show that the Earth observation missions have been operated successfully with respect to that the meteorological mission is required to be continued 24 hours a day and 365 days a year and the ocean mission is required to be operated 8 times a day and 365 days a year. And, these operational results also mean that the stability and the robustness of the mission operation are successfully verified on a top system level in all aspects of the satellite bus/payload, the ground station facilities/ equipments, and the ground operator activities in the normal operation service of the COMS.

Finally, taking into account that the COMS performs the first ocean monitoring mission in the world and the first meteorological observation mission in Korea on the geostationary Earth orbit, it is expected that the results of this paper can be a useful reference to discussion about similar future mission operation such as the COMS follow-on, too.

\section{Acknowledgements}

The COMS is operating by the cooperation of KARI, KMA, KIOST, and 'Electronics and Telecommunications Research Institute' (ETRI) under the financial supports from the Korean government ministries of 'Ministry of Science, ICT \& Future
Planning' (MSIP), KMA, and 'Ministry of Oceans and Fisheries' (MOF). Many thanks are given to their cooperation and supports. The COMS operators of the KARI SOC are appreciated highly for their efforts and passions devoted to the COMS operation.

\section{References}

Cho, Y., 2013. Earth Observation Mission Operation of COMS during In-Orbit Test, The Journal of the Korea Society of Space Technology, 8 (1): 89-100.

Cho, Y., C. Park, and B. Kim, 2012. Introduction to Real-Time Operation for COMS Normal Operation, IEICE Technical Report SANE2012 -97(2012-10) of International Conference on Space, Aeronautical and Navigation Elecronics 2012, Incheon, Republic of Korea, Oct. 10-12, Vol. 112, No.229, pp. 229-234.

Cho, Y. and H. Youn, 2006. Characteristics of COMS Meteorological Imager, Proc. of SPIE 2006 Sensors, Systems, and Next-Generation Satellites $X$, Stockholm, Sweden, Sep.11-13, Vol. 6361, pp. 63611G-1 - 63611G-8.

Faure, F., P.Coste, T. Benchetrit, G. Kang, and H. Kim, 2012. One year in orbit of the first Geostationary Ocean Colour Imager (GOCI), Proc. of International Conference on Space Optics, Ajaccio Corse, France, Oct. 9-12.

Kim, H., G. Kang, B. Ellis, M. Nam, H. Youn,F. Faure, P. Coste, and P. Servin, 2009. Geostationary Ocean Color Imager (GOCI), Overview and Prospect, Proc. of 60 International Astronautical Congress, Daejeon, Republic of Korea, Oct. 12-16, IAC-09.B1.3.9, pp. 1-11.

Kim, H., Y. Kim, Y. Cho, G. Kang, D. Lee, and Y. Chang, 2010. On COMS Optical Sensors and Their Applications, Proc. of 2010 International Symposiumon Remote Sensing / 2010 International 
Conference on Space, Aeronautical and Navigational Electronics, Jeju, Republic of Korea, Oct. 2729, pp. 271-274.

Lee, S., B. Park, B. Kim, G. Ju, and Y. Park, 2006. A Study on the East/West Station Keeping Planning Considering Wheel Off-Loading, Journal of the Korean Society for Aeronautical and Space Sciences, 34(9): 60-66 (In Korean with English abstract).

Lee, S., J. Jo, M. You, J. Choi, and K. Ahn, 2010a. Ka band Communication Payload System Technology of COMS, The Journal of the Korea Society of Space Technology, 5(2): 75-81.

Lee, S., Y. Cho, Y. Park, B. Park, B. Kim, K. Yang, and Y. Chun, 2010b. An Introduction of the N/S Station Keeping Operations for the COMS, Proc. of the 2010 KSAS Fall Conference, Jeju, Republic of Korea, Nov. 11-12,pp. 1068-1071 (In Korean with English abstract).
Lee, B., I. Kim, S. Lee, Y. Hwang, W. Jung, J. Kim, H. Kim, H. Lee, S. Lee, Y. Cho, and B. Kim, 2011a. Operational Validation of the COMS Satellite Ground Control System during the First Three Months of In-Orbit Test Operations, The Journal of the Korea Society of Space Technology, 6(1): 37-44.

Lee, B., Y. Hwang, H. Kim, and S. Park, 2011b. EastWest Station-Keeping maneuver strategy for COMS satellite using iterative process, Advances in Space Research, 47(1): 149-159.

Park, B., Y. Park, and S. Lee, 2006. COMS Momentum Dumping Optimal Thruster Set Selection, Journal of the Korean Society for Aeronautical and Space Sciences, 34(11): 54-60 (In Korean with English abstract). 